\title{
$\mathrm{MF}$ 기반 다중 사용자 Massive MIMO 시스템의 최적 기지국 안테나 수 및 사용자 수 분석
}

정 민 채*, 최 수 용

\section{Optimal Number of Base Station Antennas and Users in MF Based Multiuser Massive MIMO Systems}

\author{
Minchae Jung*, Sooyong Choi ${ }^{*}$ \\ 요 약
}

본 논문은 다중 사용자 (multiuser) 다중 안테나 (MIMO, multiple-input and multiple-output) 시스템을 기반으로 거대 안테나 시스템 (massive MIMO system)에 대한 성능 분석을 진행한다. 하향 링크 프레임 구조를 고려한 평 균 셀 용량을 도출하고, 해당 평균 셀 용량을 기지국 안테나 수 및 사용자 수에 대하여 분석한다. 평균 셀 용량은 기지국 안테나 수 및 사용자 수에 대해 오목 함수 (concave function)이며 이러한 특징을 통해 최적의 기지국 안 테나 수 및 사용자 수를 도출한다. 실험 결과를 통해 수식적으로 도출한 최적 안테나 수 및 사용자 수는 실험을 통한 최적 값과 일치함을 확인하였으며 도출한 최적 값을 통해 최대 값의 평균 셀 용량을 얻을 수 있음을 확인할 수 있다.

Key Words : Massive MIMO, Ergodic Capacity, Matched Filter (MF)

\section{ABSTRACT}

In this paper, we analyze a performance of multiuser massive multiple-input and multiple-output (MIMO) system. We derive the ergodic cell capacity based on a downlink frame structure and analyze the ergodic cell capacity with respect to the number of base station (BS) antennas and the number of users. This paper shows that the ergodic cell capacity is a concave function with respect to the number of BS antennas and the number of users, and also derives the optimal numbers of BS antennas and users for the maximum cell capacity. The simulation results verify the derived analyses and show that the derived numbers of BS antennas and users provide the maximum cell capacity.

\section{I. 서 론}

무선 통신 환경에서 사용되는 시간과 주파수 자 원은 제한적이며 그 사용에 대해 제약이 있다. 시간 과 주파수 같은 기존 자원의 제약에서 벗어나 공간 자원을 활용하는 다중 안테나 시스템 (MIMO, multiple-input and multiple-output)은 이러한 제약
을 벗어날 수 있기 때문에 무선 통신 분야에서 많 은 주목을 받고 있다 ${ }^{[1,2]}$. 이러한 다수의 공간 자원 을 다수의 사용자들에게 할당하는 다중 사용자 (multiuser) MIMO 시스템은 최근 활발히 연구되고 있는 분야 중의 하나이다 ${ }^{[3-7]}$. 나아가 공간 자원을 최대한 활용하기 위해 다수의 기지국 안테나를 활 용하는 거대 안테나 시스템 (massive MIMO)이 차

※ 이 논문은 2013년도 정부(교육과학기술부)의 재원으로 한국연구재단의 지원을 받아 수행된 기초연구사업임(no.2012R1A1A2041906).

- 주저자 : 연세대학교 전기전자공학과, hosaly@yonsei.ac.kr, 정회원

* 연세대학교 전기전자공학과, csyong@yonsei.ac.kr, 종신회원

논문번호 : KICS2013-06-259, 접수일자 : 2013년 6월 17일, 최종논문접수일자 : 2013년 8월 19일 
세대 무선 통신 기술의 핵심 기술 중 하나로 주목 받고 있다 ${ }^{[8-15]}$.

Massive MIMO 시스템은 기본적으로 다수의 기 지국 안테나 특징을 활용한다. 다수의 기지국 안테 나를 통해 주파수 효율 및 링크 신뢰도를 향상시킬 수 있으며 배열 이득 (array gain) 을 통한 커버리 지 확대의 효과 또한 얻을 수 있다 ${ }^{[8,9]}$. 하지만 massive MIMO 시스템에서는 다수의 안테나 간 간 섭 신호로 인한 간섭 문제가 발생한다 ${ }^{[6-9]}$. 하지만 다수의 기지국 안테나 특성을 기반으로 massive MIMO 시스템은 point-to-point 링크 환경에서 간단 한 MF (matched filter) 기법 및 MRC (maximum ratio combining) 기법을 통해 이상적인 채널 용량 을 얻을 수 있다는 장점이 있다 ${ }^{[8]}$. Point-to-point 링 크의 관점에서 벗어나 다중 안테나 구조를 고려할 경우에도 massive $\mathrm{MIMO}$ 시스템은 간단한 $\mathrm{MF}$ 와 $\mathrm{MRC}$ 를 통해 채널 용량을 얻을 수 있다 ${ }^{[8]}$. 또한 무 한한 기지국 안테나 수를 가정하여 $\mathrm{MF}$ 와 $\mathrm{MRC}$ 를 적용한 평균 셀 용량을 기지국 안테나의 수 및 사 용자 수를 통한 간단한 수식으로 표현할 수 있는 장점이 있다 ${ }^{[8]}$.

본 논문에서는 단일 셀 환경에서 단일 기지국이 다수의 사용자에게 신호를 전송하는 다중 사용자 $\mathrm{MIMO}$ 시스템을 고려한다. 기지국에서 사용자에게 신호를 전송하는 하향 링크 (downlink)를 고려하여 $\mathrm{MF}$ 기반의 신호를 전송하며, 매우 많은 수의 기지 국의 안테나를 고려하여 massive MIMO 시스템을 구성한다. 본 논문에서는 $\mathrm{MF}$ 기반 massive MIMO 시스템에서 OFDM (orthogonal frequency division multiplexing) 하향 링크 프레임 구조를 고려한 평 균 셀 용량을 분석하고, 이를 최대화하는 기지국 안 테나 수 및 셀 내 사용자 수를 도출한다. 또한 최 대 셀 용량의 상계 값 (upper bound)을 도출하여 $\mathrm{MF}$ 를 사용하는 massive MIMO 시스템의 한계값에 대한 분석을 진행한다.

본 논문의 구성은 다음과 같다. 2장에서는 $\mathrm{MF}$ 기반 다중 사용자 massive MIMO 시스템 모델을 정의한다. 3장에서는 기본적인 massive MIMO 시 스템의 특징에 대해서 서술하였으며 4장에서는 $\mathrm{MF}$ 기반 평균 셀 용량을 최대화하기 위한 최적 기지국 안테나 수 및 사용자 수에 대한 분석을 진행한다. 5 장에서는 제안한 최적 값에 대한 성능을 실험 결과 를 통해 보이고, 6장의 결론으로 끝을 맺는다.

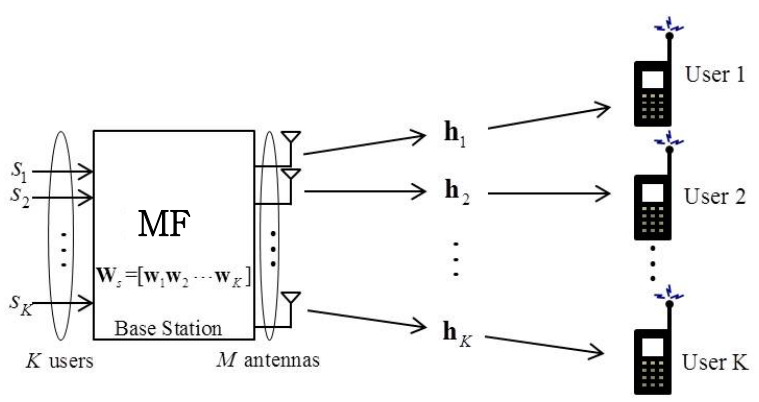

그림 1. MF 기반 다중 사용자 MIMO 시스템

Fig. 1. MF based multi-user MIMO system

\section{II. 시스템 모델}

본 논문에서는 그림 1 과 같이 단일 셀 환경에서 $M$ 개의 안테나를 가진 기지국과 한 개의 안테나를 가진 $K$ 명의 사용자로 이루어진 시스템을 고려한다. $K$ 명의 사용자는 동시에 기지국으로부터 하향 링크 신호를 수신하는 다중 사용자 MIMO 시스템을 가 정하며, 각 사용자 별 신호를 구분하기 위해서 기지 국에서는 $\mathrm{MF}$ 를 적용하여 신호를 전송한다 ${ }^{[8,9]}$. 그림 1 과 같이 해당 $\mathrm{MF}$ 행렬은 다음과 같다.

$$
\mathbf{W}_{\mathrm{S}}=\left[\mathbf{w}_{1} \mathbf{w}_{2} \cdots \mathbf{w}_{K}\right]
$$

여기서 $\mathbf{w}_{k}$ 는 $k$ 번째 사용자의 $\mathrm{MF}$ 벡터로써 $M \times 1$ 의 크기를 가지며, 전체 $\mathrm{MF}$ 행렬 $\mathbf{W}_{\mathrm{S}}$ 는 $M \times K$ 의 크기를 가진다. 또한, 기지국에서는 최대 $M$ 개의 데이터 스트림을 보낼 수 있다고 가정하여 $M \geq K$ 이다 ${ }^{[3-7]}$. 기지국으로부터 $k$ 번째 사용자로의 채널 $\mathbf{h}_{k}$ 의 모든 원소들은 평균이 0 이고 분산이 1 인 i.i.d. (independent and identically distributed) 복소 가우 시안 랜덤 변수 (complex Gaussian random variable)를 고려한다. 따라서 모든 사용자에 대한 전체 채널 $\mathbf{H}_{\mathrm{S}}$ 는 $K \times M$ 크기를 가지며 다음과 같다.

$$
\mathbf{H}_{\mathrm{S}}=\left[\mathbf{h}_{1}^{T} \mathbf{h}_{2}^{T} \cdots \mathbf{h}_{K}^{T}\right]^{T}
$$

본 논문에서는 기지국에서 $\mathbf{H}_{\mathrm{S}}$ 에 대한 정보를 완 벽하게 알고 있다고 가정하며, 따라서 $\mathrm{MF}$ 행렬 $\mathbf{W}_{\mathrm{S}}$ 는 다음과 같다 ${ }^{[8,9]}$.

$$
\mathbf{W}_{\mathrm{S}}=\left(\mathbf{H}_{\mathrm{S}}\right)^{H}
$$

여기서 $\mathbf{A}^{\mathrm{H}}$ 는 $\mathbf{A}$ 행렬에 대한 conjugate transpose를 의미한다. 기지국에서 해당 $\mathrm{MF}$ 를 적용 하여 신호를 전송하는 경우, $k$ 번째 사용자의 수신 신호는 다음과 같다. 


$$
\begin{aligned}
y_{k} & =\sqrt{P_{\mathrm{BS}}} \mathbf{h}_{k} \frac{\mathbf{w}_{k}}{\left\|\mathbf{W}_{\mathrm{S}}\right\|_{\mathrm{F}}} s_{k}+\sum_{i=1, i \neq k}^{K} \sqrt{P_{\mathrm{BS}}} \mathbf{h}_{k} \frac{\mathbf{w}_{i}}{\left\|\mathbf{W}_{\mathrm{S}}\right\|_{\mathrm{F}}} s_{i}+n_{k} \\
& =\sqrt{P_{\mathrm{BS}}} \frac{\left\|\mathbf{h}_{k}\right\|^{2}}{\left\|\mathbf{H}_{\mathrm{S}}\right\|_{\mathrm{F}}} s_{k}+\sum_{i=1, i \neq k}^{K} \sqrt{P_{\mathrm{BS}}} \frac{\mathbf{h}_{k} \mathbf{h}_{i}^{H}}{\left\|\mathbf{H}_{\mathrm{S}}\right\|_{\mathrm{F}}} s_{i}+n_{k}
\end{aligned}
$$

여기서 $P_{\mathrm{BS}}$ 는 기지국에서의 하향 링크 총 송신 파워이며, $s k$ 는 $k$ 번째 사용자로의 하향 링크 정보 신호로써 평균이 0 이고 분산이 1 인 독립적인 가우 시안 랜덤 변수로 가정한다. 또한 $\left\|\mathbf{W}_{\mathrm{s}}\right\|_{\mathrm{F}}$ 는 기지국에 서의 $\mathrm{MF}$ 행렬 정규화 (normalization)를 위한 성분 이며, $n k$ 는 부가 백색 가우시안 잡음 (AWGN, additive white Gaussian noise)으로써 $\mathrm{E}\left[n_{k} n_{k}^{*}\right]=N_{0}$ 의 공분산 값을 갖는다. 여기서 $\|\mathbf{A}\|_{\mathrm{F}}$ 는 $\mathbf{A}$ 행렬의 Frobenious norm을 의미한다. 본 논문에서는 식의 간소화를 위해 $P \mathrm{BS} / N_{0}=\rho$ 로로 정의한다.

\section{Massive MIMO 시스템의 특징}

기본적으로 massive $\mathrm{MIMO}$ 시스템은 다수의 기 지국 안테나를 기반으로 다음과 같은 특성을 활용 한다 ${ }^{[8]-[9]} . i$ 번째 사용자와 $j$ 번째 사용자의 채널 $\mathbf{h}_{i}$, $\mathbf{h}_{j}$ 를 다음과 같이 정의했을 때,

$$
\mathbf{h}_{i}=\left[h_{1}^{i}, h_{2}^{i}, \cdots, h_{M}^{i}\right], \mathbf{h}_{j}=\left[h_{1}^{j}, h_{2}^{j}, \cdots, h_{M}^{j}\right]
$$

무한히 많은 안테나 수 $(M)$ 에 대하여, 각 채널에 대한 크기 및 서로 다른 채널의 연산에 대한 값은 다음과 같다.

$$
\begin{array}{r}
\frac{\left\|\mathbf{h}_{i}\right\|^{2}}{M}=\frac{\left|h_{1}^{i}\right|^{2}+\cdots+\left|h_{M}^{i}\right|^{2}}{M} \approx \underbrace{\operatorname{Var}[h]}_{1}+\underbrace{(\mathrm{E}[h])^{2}}_{0}=1 \\
\frac{\mathbf{h}_{i} \mathbf{h}_{j}^{H}}{M}=\frac{1}{M}\left(h_{1}^{i} h_{1}^{j^{*}}+h_{2}^{i} h_{2}^{j^{*}}+\cdots h_{M}^{i} h_{M}^{j^{*}}\right) \\
=\frac{g_{1}+\cdots+g_{M}}{M}=\mathrm{E}[g]=(\mathrm{E}[h])^{2}=0
\end{array}
$$

여기서 랜덤 변수 $h$ 는 평균이 0 이고 분산이 1 인 i.i.d. 복소 가우시안 랜덤 변수이므로 식 (2)와 같 이 랜덤 변수 $g$ 의 평균값은 0 이다. 그림 2 에서 보 듯이 같은 채널의 연산 값은 $M$ 의 증가에 따라 1 에 수렴하며, 다른 채널의 연산 값은 $M$ 의 증가에 따라 0 에 수렴한다. 이러한 특징을 바탕으로 전체 채널 $\mathrm{H}_{\mathrm{S}}$ 의 정규화 된 크기는 다음과 같다.

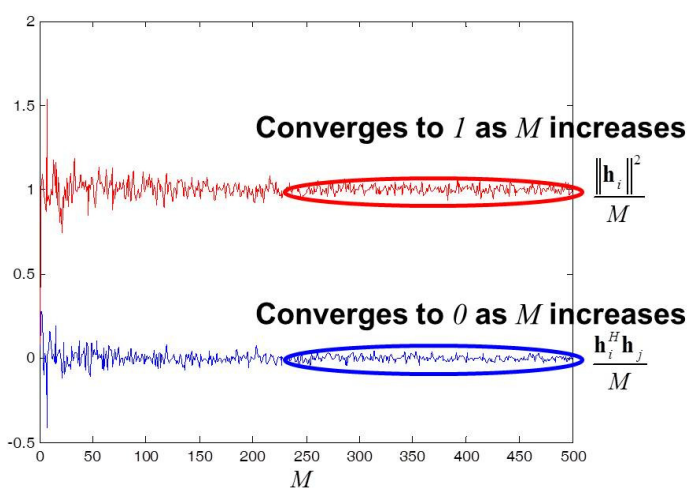

그림 2. 기본적인 massive MIMO의 특징

Fig. 2. Fundamental characteristic of massive MIMO

$$
\begin{aligned}
\frac{1}{M} \mathbf{H}_{\mathrm{S}} \mathbf{H}_{\mathrm{S}}^{H} & =\frac{1}{M}\left[\begin{array}{c}
-\mathbf{h}_{1}- \\
\vdots \\
-\mathbf{h}_{K}-
\end{array}\right]\left[\begin{array}{ccc}
\mid & & \mid \\
\mathbf{h}_{1}^{H} & \cdots & \mathbf{h}_{K}^{H} \\
\mid & & \mid
\end{array}\right] \\
& =\frac{1}{M}\left[\begin{array}{cccc}
\left\|\mathbf{h}_{1}\right\|^{2} & \mathbf{h}_{1} \mathbf{h}_{2}^{H} & \cdots & \mathbf{h}_{1} \mathbf{h}_{K}^{H} \\
\mathbf{h}_{2} \mathbf{h}_{1}^{H} & \left\|\mathbf{h}_{2}\right\|^{2} & & \\
\vdots & & \ddots & \\
\mathbf{h}_{K} \mathbf{h}_{1}^{H} & \mathbf{h}_{K} \mathbf{h}_{2}^{H} & \cdots & \left\|\mathbf{h}_{K}\right\|^{2}
\end{array}\right] \approx \mathbf{I}_{K}
\end{aligned}
$$

여기서 $\mathbf{I}_{K}$ 는 $K \times K$ 크기의 항등 행렬 (identity matrix)를 나타낸다. 이러한 기본적인 특성을 바탕 으로, 다중 안테나 massive MIMO 하향 링크의 환 경에서 사용자가 기지국처럼 모든 채널에 대한 정 보를 알고 있다고 가정하였을 경우의 평균 셀 용량 (ergodic cell capacity)은 다음과 같다 ${ }^{[8]}$.

$$
\begin{aligned}
C_{\mathrm{DL}} & =\log _{2} \operatorname{det}\left(\mathbf{I}_{K}+\frac{\rho_{\mathrm{d}}}{K} \mathbf{H}_{\mathrm{S}} \mathbf{H}_{\mathrm{S}}^{H}\right)=\log _{2} \operatorname{det}\left(\mathbf{I}_{K}+M \frac{\rho_{\mathrm{d}}}{K} \frac{\mathbf{H}_{\mathrm{S}} \mathbf{H}_{\mathrm{S}}^{H}}{M}\right) \\
& \approx \log _{2} \operatorname{det}\left(\mathbf{I}_{K}+\frac{M}{K} \rho_{\mathrm{d}_{K}} \mathbf{I}_{K}\right)=K \log _{2}\left(1+\frac{M}{K} \rho_{\mathrm{d}}\right)
\end{aligned}
$$

여기서 식 (4)는 식 (3)을 이용하여 전개된다. 시 스템 모델에서 고려한 바와 같이 기지국에서 $\mathrm{MF}$ 를 적용한 경우, 수신 신호는 식 (1)과 같이 표현되며, 여기서 식 (1)의 정규화 부분은 식 (2)를 통해 다음 과 같다.

$$
\left\|\mathbf{H}_{\mathrm{S}}\right\|_{\mathrm{F}}=\sqrt{\left\|\mathbf{h}_{1}\right\|^{2}+\cdots+\left\|\mathbf{h}_{K}\right\|^{2}} \approx \sqrt{M K}
$$

따라서, 무한히 큰 수의 $M$ 에 대해, 식 (2)와 식 (5)를 바탕으로 식 (1)은 다음과 같다. 


$$
\begin{aligned}
y_{k} & =\sqrt{P_{\mathrm{BS}}} \frac{\left\|\mathbf{h}_{k}\right\|^{2}}{\left\|\mathbf{H}_{\mathrm{S}}\right\|_{\mathrm{F}}} s_{k}+\sum_{i=1, i \neq k}^{K} \sqrt{P_{\mathrm{BS}}} \frac{\mathbf{h}_{k} \mathbf{h}_{i}^{H}}{\left\|\mathbf{H}_{\mathrm{S}}\right\|_{\mathrm{F}}} s_{i}+n_{k} \\
& \approx \sqrt{\frac{P_{\mathrm{BS}}}{M K} \frac{\left\|\mathbf{h}_{k}\right\|^{2}}{M}} M s_{k}+\sum_{i=1, i \neq k}^{K} \sqrt{\frac{P_{\mathrm{BS}}}{M K}} \frac{\mathbf{h}_{k} \mathbf{h}_{i}^{H}}{M} M s_{i}+n_{k} \\
& \approx \sqrt{M P_{\mathrm{BS}} / K} s_{k}+n_{k}
\end{aligned}
$$

따라서 $\mathrm{MF}$ 적용 후의 사용자 별 수신 $\mathrm{SNR}$ (signal to noise ratio)은 다음과 같다.

$$
\gamma_{\mathrm{d}}=\frac{M}{K} \frac{P_{\mathrm{BS}}}{N_{0}}=\frac{M}{K} \rho_{\mathrm{d}}
$$

식 (4)와 식 (7)을 통하여, 다중 사용자 massive $\mathrm{MIMO}$ 시스템에서의 하향 링크 평균 셀 용량은 간 단한 MF를 통해 달성할 수 있음을 확인할 수 있다.

상향 링크의 경우 다중 사용자 massive $\mathrm{MIMO}$ 환경에서의 전체 채널 $\mathrm{Gs}$ 는 $M \times K$ 의 크기를 가지며 다음과 같다. $\mathbf{G}_{\mathrm{S}}=\left[\mathbf{g}_{1} \mathbf{g}_{2} \cdots \mathbf{g}_{K}\right]$ 여기서 $\mathbf{g}_{k}$ 는 크기 $M \times 1$ 을 갖는 $k$ 번째 사용자의 상향 링크 채널이다. 다중 사용자 massive MIMO 환경에서 사용자들끼 리 협력을 하여 정보 교환을 한다고 가정하였을 경 우의 평균 셀 용량은 다음과 같다.

$$
\begin{aligned}
C_{\mathrm{UL}} & =\log _{2} \operatorname{det}\left(\mathbf{I}_{K}+\rho_{\mathrm{u}} \mathbf{G}_{\mathrm{S}}^{H} \mathbf{G}_{\mathrm{S}}\right)=\log _{2} \operatorname{det}\left(\mathbf{I}_{K}+M \rho_{\mathrm{u}} \frac{\mathbf{G}_{\mathrm{S}}^{H} \mathbf{G}_{\mathrm{S}}}{M}\right) \\
& \approx \log _{2} \operatorname{det}\left(\mathbf{I}_{K}+M \rho_{\mathrm{u}} \mathbf{I}_{K}\right)=K \log _{2}\left(1+M \rho_{\mathrm{u}}\right)
\end{aligned}
$$

여기서 $\rho_{\mathrm{u}}=P_{\mathrm{UE}} / N_{0}$ 는 상향 링크 송신 $\mathrm{SNR}$, $P \mathrm{UE}$ 는 사용자의 상향 링크 송신 파워를 의미한다. 다중 사용자 $\mathrm{MIMO}$ 환경의 상향 링크에서, 중첩된 다수의 상향 링크 사용자 신호를 구분하기 위해 기 지국에서는 $\mathrm{MRC}$ 를 적용한다 ${ }^{[8]}$. 무한히 큰 수의 기 지국 안테나 수 $(M)$ 에 대해, $\mathrm{MRC}$ 를 적용한 후 $k$ 번째 사용자의 수신 신호는 다음과 같다.

$$
\begin{aligned}
y_{k} & =\sqrt{P_{\mathrm{UE}}}\left\|\mathbf{g}_{k}\right\|^{2} s_{k}+\sum_{i=1, i \neq k}^{K} \sqrt{P_{\mathrm{UE}}} \mathbf{g}_{k}^{H} \mathbf{g}_{i} s_{i}+\mathbf{g}_{k}^{H} \mathbf{n}_{k} \\
& \approx \sqrt{P_{\mathrm{UE}}} M s_{k}+\mathbf{g}_{k}^{H} \mathbf{n}_{k}
\end{aligned}
$$

따라서 $\mathrm{MRC}$ 적용 후의 사용자 별 수신 $\mathrm{SNR}$ 은 다음과 같다.
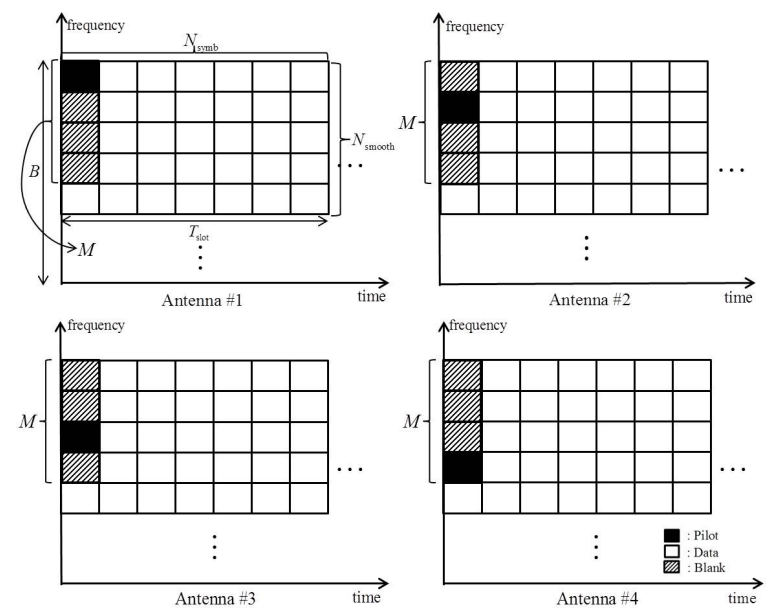

그림 3. 기지국 안테나가 4 인 경우의 $\mathrm{OFDM}$ 기반 하향 링 크 프레임 구조

Fig. 3. OFDM based downlink frame structure when $M=$

$$
\gamma_{\mathrm{d}}=\frac{M^{2} P_{\mathrm{UE}}}{\left|\mathbf{g}_{k}^{H} \mathbf{n}_{k}\right|^{2}} \approx \frac{M^{2} P_{\mathrm{UE}}}{M N_{0}}=M \rho_{\mathrm{u}}
$$

식 (8)와 식 (10)을 통하여, 다중 사용자 massive $\mathrm{MIMO}$ 시스템에서의 상향 링크 평균 셀 용량 또한 간단한 $\mathrm{MRC}$ 를 통해 달성할 수 있음을 확인할 수 있다.

\section{IV. 평균 셀 용량 최대화를 위한 최적 안테나 수 및 사용자 수 분석}

기지국에서 $M$ 개의 안테나를 사용하는 경우, 3GPP Long Term Evolution (LTE) 시스템에서는 $M$ 개의 안테나 별 독립적인 자원에 파일럿 신호를 전송한다 ${ }^{[16]}$. 그림 3의 OFDM 기반 하향 링크 프레 임 구조와 같이 기지국에서는 독립된 시공간 자원 에 파일럿을 전송하고 각 사용자들은 해당 파일럿 신호를 통해 안테나 별 채널을 추정한다. 여기서 주 파수 축 채널 응답은 $N_{\text {smooth }}$ 동안 동일한 값을 가 지며 시간 축 채널 응답은 $T_{\text {slot }}$ 동안 동일한 값을 가짐을 가정한다 ${ }^{[9]}$. 그림 3 의 프레임 구조를 가정하 여 $\mathrm{MF}$ 를 적용한 하향 링크 평균 셀 용량은 다음과 같다 ${ }^{[8]}$.

$$
\begin{aligned}
R_{\mathrm{MF}} & =\mathrm{E}\left[\sum_{k=1}^{K} \frac{B T_{\mathrm{U}}}{T_{\mathrm{S}}}\left(1-\frac{M}{N_{\mathrm{SS}}}\right) \log _{2}\left(1+\mathrm{SINR}_{k}\right)\right] \\
& =\frac{B T_{\mathrm{U}}}{T_{\mathrm{S}}}\left(1-\frac{M}{N_{\mathrm{SS}}}\right) \mathrm{E}\left[\sum_{k=1}^{K} \log _{2}\left(1+\mathrm{SINR}_{k}\right)\right]
\end{aligned}
$$


여기서 $N_{\mathrm{SS}}=N_{\mathrm{symb}} N_{\mathrm{smooth}}$ 이며, $T_{\mathrm{S}}$ 와 $T_{\mathrm{U}}$ 는 각각 전 체 심볼 시간과 실제 데이터가 전송되는 시간을 의 미하고 $B$ 는 하향 링크 시스템 대역폭 (bandwidth) 을 의미한다. $N_{\mathrm{symb}}$ 는 $T_{\mathrm{slot}}$ 동안의 $\mathrm{OFDM}$ 심볼 수 를 의미한다. $\operatorname{SINR}_{k}$ 는 $k$ 번째 사용자의 수신 SINR (signal to interference plus noise ratio)를 나타내 며, massive MIMO 환경에서 $\mathrm{MF}$ 를 사용하였을 경 우 각 사용자의 수신 SINR은 다음과 같다 ${ }^{[8]}$.

$$
\operatorname{SINR}_{k}=\frac{\rho_{\mathrm{d}} M}{K\left(\rho_{\mathrm{d}}+1\right)}
$$

따라서 모든 사용자의 수신 SINR은 사용자에 상 관없이 모두 동일한 값을 지님을 확인할 수 있다. 식 (12)를 통해 식 (11)은 다음과 같다.

$$
\begin{aligned}
R_{\mathrm{MF}} & =\frac{B T_{\mathrm{U}}}{T_{\mathrm{S}}}\left(1-\frac{M}{N_{\mathrm{SS}}}\right) \mathrm{E}\left[\sum_{k=1}^{K} \log _{2}\left(1+\frac{\rho_{\mathrm{d}} M}{K\left(\rho_{\mathrm{d}}+1\right)}\right)\right] \\
& =\frac{K B T_{\mathrm{U}}}{T_{\mathrm{S}}}\left(1-\frac{M}{N_{\mathrm{SS}}}\right) \sum_{k=1}^{K} \log _{2}\left(1+\frac{\rho_{\mathrm{d}} M}{K\left(\rho_{\mathrm{d}}+1\right)}\right)
\end{aligned}
$$

따라서 평균 셀 용량 최대화를 위한 사용자 수 및 안테나 수에 대한 최적화 문제는 다음과 같다.

$$
\begin{aligned}
& \max _{K, M} \frac{K B T_{\mathrm{U}}}{T_{\mathrm{S}}}\left(1-\frac{M}{N_{\mathrm{SS}}}\right) \log _{2}\left(1+\frac{\rho_{\mathrm{d}} M}{K\left(\rho_{\mathrm{d}}+1\right)}\right) \\
& \text { s.t. } 0 \leq K \leq M \leq N_{\mathrm{SS}}
\end{aligned}
$$

식 (14)에서 사용자 수 $K$ 와 안테나 수 $M$ 은 서로 독립적인 변수이기 때문에 각 변수에 대한 독립적 인 분석을 통해 문제를 해결할 수 있다. 식 (14)에 나타낸 목적 함수 (objective function)의 $K$ 에 대한 특성을 파악하기 위해, 목적 함수를 $K$ 에 대한 1 차 미분을 진행하면 다음과 같다.

$$
\frac{\partial R_{\mathrm{MF}}}{\partial K}=\frac{B T_{\mathrm{U}}\left(N_{\mathrm{SS}}-M\right)\left(\left(K\left(1+\rho_{\mathrm{d}}\right)+M \rho_{\mathrm{d}}\right) \ln \left(1+\frac{M \rho_{\mathrm{d}}}{K\left(1+\rho_{\mathrm{d}}\right)}\right)-M \rho_{\mathrm{d}}\right)}{T_{\mathrm{S}} N_{\mathrm{SS}} \ln 2\left(K+\rho_{\mathrm{d}}(M+K)\right)}
$$

$N_{\mathrm{Ss}} \geq M$ 이므로, 식 (15)에 따라서 $R_{\mathrm{MF}}$ 가 $K$ 에 대 해 단조 증가할 조건은 다음과 같다.

$$
\begin{gathered}
(K(1+\rho)+M \rho) \ln \left(1+\frac{M \rho}{K(1+\rho)}\right) \geq M \rho \\
\Leftrightarrow \frac{K(1+\rho)+M \rho}{K(1+\rho)} \geq e^{\frac{M \rho}{(K(1+\rho)+M \rho)}} \\
\Leftrightarrow \frac{K(1+\rho)+M \rho}{e K(1+\rho)} \geq e^{\frac{-K(1+\rho)}{(K(1+\rho)+M \rho)}} \\
\Leftrightarrow-\frac{1}{e} \leq \frac{-K(1+\rho)}{K(1+\rho)+M \rho} e^{\frac{-K(1+\rho)}{(K(1+\rho)+M \rho)}} \\
\stackrel{(a)}{\Leftrightarrow} \operatorname{LW}\left(-\frac{1}{e}\right) \leq \frac{-K(1+\rho)}{K(1+\rho)+M \rho}
\end{gathered}
$$

여기서 $\mathrm{LW}(X)$ 는 Lambert-W 함수이며 $Y=\mathrm{LW}(X)$ 는 $Y e^{Y}=X$ 의 솔루션을 의미한다 ${ }^{[17]}$. 또한 $\mathrm{LW}(X)$ 함 수는 $X \geq-1 / e$ 영역에서 단조 증가 함수이기 때문에 (a)가 성립한다 ${ }^{[17]} \cdot \mathrm{LW}(-1 / e)=-1$ 이기 때문에 식 (16) 은 다음과 같이 전개된다.

$$
-1 \leq \frac{-K(1+\rho)}{K(1+\rho)+M \rho} \Leftrightarrow 1 \leq 1+\frac{M \rho}{K(1+\rho)}
$$

양수인 $M, K, \rho$ 에 대해 식 (17)은 항상 성립하 기 때문에, 결과적으로 식 (14)의 목적 함수는 $K$ 에 대해 고려하는 모든 영역에서 단조 증가 함수이다. 따라서 $R_{\mathrm{MF}}$ 를 최대화 하는 $K$ 는 다음과 같다.

$$
K_{\text {opt }}=M
$$

따라서 식 (14)는 다음과 같이 재구성 된다.

$$
\begin{aligned}
& \max _{M} \frac{M B T_{\mathrm{U}}}{T_{\mathrm{S}}}\left(1-\frac{M}{N_{\mathrm{SS}}}\right) \log _{2}\left(1+\frac{\rho_{\mathrm{d}}}{\rho_{\mathrm{d}}+1}\right) \\
& \text { s.t. } 0 \leq M \leq N_{\mathrm{SS}}
\end{aligned}
$$

식 (19)의 목적 함수의 오목 형태(concavity)를 판단하기 위해 목적 함수의 2 차 미분을 진행한다.

$$
\frac{\partial^{2} R_{\mathrm{MF}}}{\partial M^{2}}=\frac{-2 B T_{\mathrm{U}}}{N_{\mathrm{SS}} T_{\mathrm{S}} \ln 2} \ln \left(1+\frac{\rho_{\mathrm{d}}}{1+\rho_{\mathrm{d}}}\right) \leq 0
$$

식 (20)은 항상 음수를 갖기 때문에 식 (19)의 목적 함수는 오목 함수이다. 따라서 최적의 안테나 수 $(M)$ 는 다음과 같이 목적 함수의 1 차 미분이 0 이 되는 $M$ 값 이다. 


$$
\frac{\partial R_{\mathrm{MF}}}{\partial M}=\frac{B\left(N_{\mathrm{SS}}-2 M\right) T_{\mathrm{U}} \ln \left(1+\frac{\rho_{\mathrm{d}}}{1+\rho_{\mathrm{d}}}\right)}{N_{\mathrm{SS}} T_{\mathrm{S}} \ln 2}=0
$$

따라서 $R_{\mathrm{MF}}$ 를 최대화 하는 $M$ 은 다음과 같다.

$$
M_{\mathrm{opt}}=\frac{N_{\mathrm{SS}}}{2}
$$

식 (18)과 식 (22)를 통하여, 최적화 문제 식 (14)의 해는 다음과 같다.

$$
M_{\mathrm{opt}}=K_{\mathrm{opt}}=\frac{N_{\mathrm{SS}}}{2}
$$

결론적으로 $\mathrm{MF}$ 를 사용하는 massive $\mathrm{MIMO}$ 하 향 링크의 경우, 최적의 사용자 수 및 안테나 수는 동일하게 나타나며 그 값은 시간과 주파수 축으로 채널 상태가 유지되는 부 반송파(subcarrier)의 개수 의 절반에 해당된다. 따라서 주파수 비 선택적이며 (frequency flat) 시간 불변의 (time invariant) 채널 환경일수록 더 많은 사용자 수와 안테나 수를 최적 의 값으로 요구한다. 반면에 주파수 선택적이며 (frequency selective) 시간에 따라 급변하는 (time varying) 채널의 경우 최적의 사용자 수 및 안테나 수는 작은 값을 가지게 되며, 이는 massive MIMO 의 가정에 위배된다. 따라서 식 (23)의 분석 결과는 채널 상태가 어느 정도 시간과 주파수 축으로 유지 되어 $M_{\mathrm{opt}}$ 값이 큰 값을 갖는 상황에서 의미가 있 음을 확인할 수 있다.

식 (23)을 통해 $\mathrm{MF}$ 기반의 최적 셀 용량은 에 대입하여 다음을 얻을 수 있다.

$$
R_{\mathrm{MF}}^{\mathrm{MAX}}=\frac{N_{\mathrm{SS}} B T_{\mathrm{U}}}{4 T_{\mathrm{S}}} \log _{2}\left(1+\frac{\rho_{\mathrm{d}}}{\rho_{\mathrm{d}}+1}\right)
$$

따라서 $\mathrm{MF}$ 기반 최적 셀 용량은 $N_{\mathrm{ss}}$ 가 증가함에 따라 선형적으로 증가한다. 또한, 식 (24)를 하향 링크 송신 $\operatorname{SNR}\left(\rho_{\mathrm{d}}\right)$ 에 관하여 1 차, 2 차 미분을 하 면 각각 다음과 같다.

$$
\begin{aligned}
& \frac{\partial R_{\mathrm{MF}}^{\mathrm{MAX}}}{\partial \rho_{\mathrm{d}}}=\frac{N_{\mathrm{SS}} B T_{\mathrm{U}}}{4 T_{\mathrm{S}}\left(\ln 2+\rho_{\mathrm{d}} \ln 8+\rho_{\mathrm{d}}^{2} \ln 4\right)} \geq 0 \\
& \frac{\partial^{2} R_{\mathrm{MF}}^{\mathrm{MAX}}}{\partial \rho_{\mathrm{d}}^{2}}=\frac{-N_{\mathrm{SS}} B T_{\mathrm{U}}\left(3+4 \rho_{\mathrm{d}}\right)}{4 T_{\mathrm{S}} \ln 2\left(1+3 \rho_{\mathrm{d}}+2 \rho_{\mathrm{d}}^{2}\right)^{2}} \leq 0
\end{aligned}
$$

식 (25)를 통해 식 (24)는 오목 함수 (concave function)의 형태를 가지고 있으며 $\rho_{\mathrm{t}}$ 의 증가에 따 라 단조 증가하는 형태의 함수이다.

표 1. 실험 환경

Table 1. Simulation parameters

\begin{tabular}{|c|c|}
\hline Parameter & Value \\
\hline System bandwidth $(B)$ & $10 \mathrm{MHz}$ \\
\hline Coherence time & 500 us \\
\hline $\begin{array}{c}\text { Time duration } \\
\text { for a time slot }\left(T_{\text {slot }}\right)\end{array}$ & 500 us \\
\hline $\begin{array}{c}\text { Symbol interval }\left(T_{\mathrm{S}}\right) \\
\text { Useful symbol duration } \\
\left(T_{\mathrm{U}}\right)\end{array}$ & 71.4 us \\
\hline $\begin{array}{c}\text { Frequency flat interval } \\
\left(N_{\text {smooth }}\right)\end{array}$ & 30 subcarriers \\
\hline $\begin{array}{c}\text { \# of OFDM symbols } \\
\text { in a time slot }\left(N_{\text {symb }}\right)\end{array}$ & 7 OFDM symbols \\
\hline
\end{tabular}

하지만 무한 값의 $\rho_{\mathrm{d}}$ 에 대해서는 1 차 미분 결과 가 0 으로 수렴하게 되고 이는 $\mathrm{MF}$ 기반 최적 셀 용 량이 특정 값으로 수렴하게 됨을 의미한다. 무한 값 의 $\rho_{\mathrm{d}}$ 를 가정하여 식 (24)를 전개하면 다음과 같 다.

$$
\begin{aligned}
\lim _{\rho_{\mathrm{d}} \rightarrow \infty} R_{\mathrm{MF}}^{\mathrm{MAX}} & =\lim _{\rho_{\mathrm{d}} \rightarrow \infty} \frac{N_{\mathrm{SS}} B T_{\mathrm{U}}}{4 T_{\mathrm{S}}} \log _{2}\left(1+\frac{\rho_{\mathrm{d}}}{\rho_{\mathrm{d}}+1}\right) \\
& =\frac{N_{\mathrm{SS}} B T_{\mathrm{U}}}{4 T_{\mathrm{S}}}
\end{aligned}
$$

$\mathrm{MF}$ 기반 최적 셀 용량은 $\rho_{\mathrm{d}}$ 에 의해 단조 증가 하며 무한한 $\rho_{\mathrm{d}}$ 에서 $N_{\mathrm{SS}} B T_{\mathrm{U}} / 4 T_{\mathrm{S}}$ 에 수렴하기 때문 에, 해당 수렴 값은 $\mathrm{MF}$ 기반 최적 셀 용량의 상계 값을 의미한 다. 결론적으로 $\mathrm{MF}$ 를 통한 평균 셀 용량의 최대 값은 $N_{\mathrm{SS}} B T_{\mathrm{U}} / 4 T_{\mathrm{S}}$ 이다.

\section{V. 실험 결과}

본 장에서는 다중 사용자 massive MIMO 환경 에서의 MF 기반 셀 용량에 대한 실험을 진행하였 다. 본 장에서 사용한 실험 파라미터는 $3 \mathrm{GPP} \mathrm{LTE}$ 표준 ${ }^{16]}$ 을 기반으로 진행하였으며 표 1 과 같다. 그 림에서 표시된 'Monte Carlo'는 i.i.d.한 복소 가우 시안 랜덤 변수로 사용자 별 채널을 발생시키고 $\mathrm{MF}$ 를 적용시킨 후 셀 용량을 측정한 결과이며, 


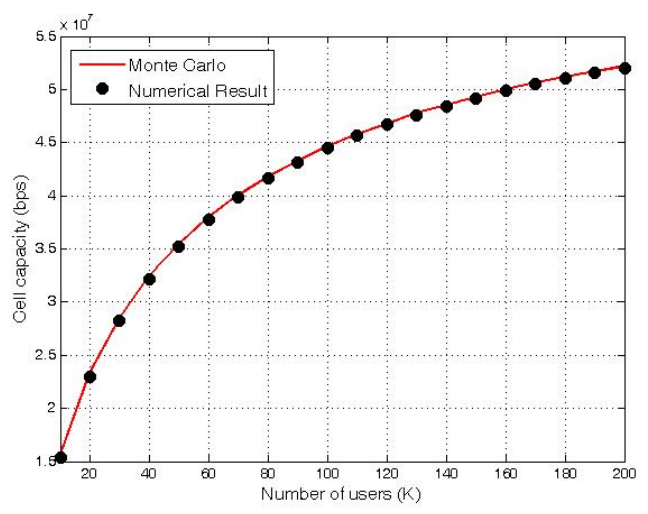

그림 4. 기지국의 송신 $\mathrm{SNR}$ 이 $0 \mathrm{~dB}$, 기지국 안테나 수가 200개일 경우의 셀 내 사용자 수에 따른 $\mathrm{MF}$ 기반 셀 용량 Fig. 4. MF based cell capacity when transmit $\mathrm{SNR}=$ $0 \mathrm{~dB}$ and $\mathrm{M}=200$

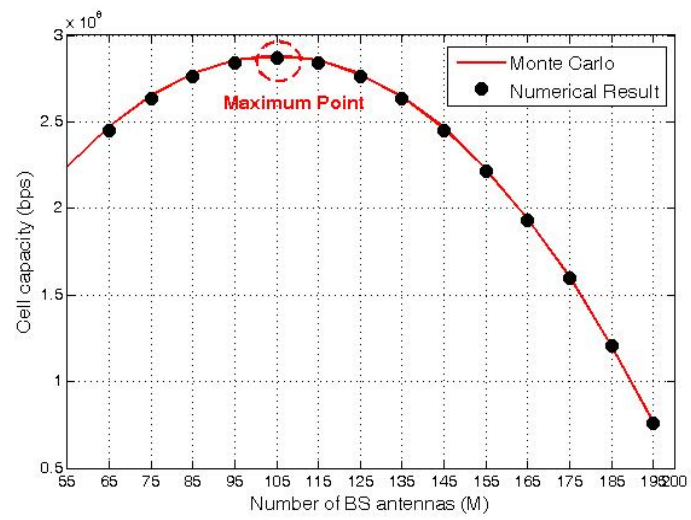

그림 5. 기지국의 송신 SNR이 $0 \mathrm{~dB}$, 셀 내 사용자 수가 $M$ 명 일 경우의 기지국 안테나 수에 따른 $\mathrm{MF}$ 기반 셀 용량 Fig. 5. MF based cell capacity when transmit SNR = $0 \mathrm{~dB}$ and $K=M$

'Numerical Result'는 식 (13)을 통한 수식을 적용 한 실험 결과를 의미한다 ${ }^{[18]}$.

그림 4는 기지국의 송신 $\mathrm{SNR}$ 이 $0 \mathrm{~dB}$, 기지국 안 테나 수가 200개일 경우의 셀 내 사용자 수에 따른 $\mathrm{MF}$ 기반 셀 용량을 보여주고 있다. 식 (17)의 분석 결과와 동일하게, $\mathrm{MF}$ 기반의 셀 용량은 셀 내 사 용자 수의 증가에 따라 증가한다. 또한 실험을 통한 실험 값과 추정한 수식은 유사한 결과를 나타내고 있음을 확인할 수 있다.

그림 5 는 기지국의 송신 $\mathrm{SNR}$ 이 $0 \mathrm{~dB}$, 셀 내 사 용자 수가 $M$ 명 일 경우의 기지국 안테나 수에 따 른 $\mathrm{MF}$ 기반 셀 용량을 보여주고 있다. 그림 4 와 마찬가지로, 실험을 통한 실험 값과 추정한 수식은 유사한 결과를 나타내고 있다. 또한 식 (20)의 분석 결과와 동일하게, $\mathrm{MF}$ 기반의 셀 용량은 기지국의 안테나 수에 따라 오목 함수 형태를 보이고 있음을 확인할 수 있다. 따라서 그림 5 에 표시된 바와 같

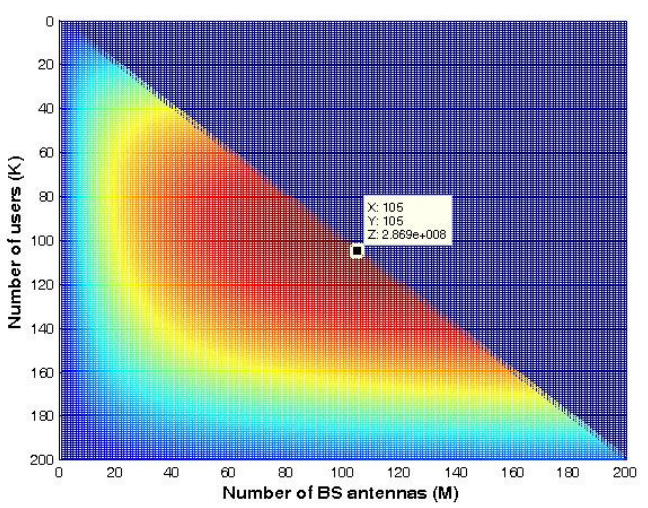

그림 6. 기지국의 송신 $\mathrm{SNR}$ 이 $0 \mathrm{~dB}$ 일 경우의 기지국 안테나 수 및 셀 내 안테나 수에 따른 MF 기반 셀 용량

Fig. 6. MF based cell capacity when transmit SNR = $0 \mathrm{~dB}$

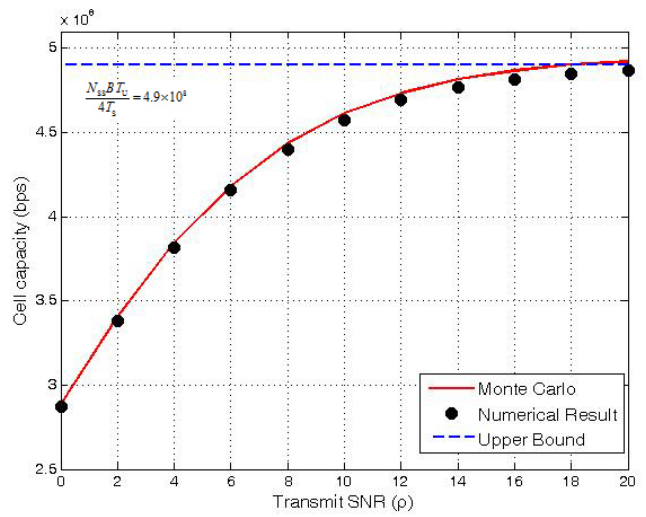

그림 7. 최적 기지국 안테나 수 및 사용자 수 기반의 기지 국 송신 SNR에 따른 셀 용량

Fig. 7. MF based cell capacity for optimal $M$ and $K$

이 $\mathrm{MF}$ 기반 셀 용량의 최대 값이 존재하며 그 값 은 전수 조사 실험을 통해 105이다. 식 (22)를 통해 최적 기지국 안테나 수는 $N_{\mathrm{ss}} / 2=105$ 이며 이는 실험을 통한 결과와 동일함을 확인할 수 있다.

그림 6은 기지국의 송신 $\mathrm{SNR}$ 이 $0 \mathrm{~dB}$ 일 경우의 기지국 안테나 수 및 셀 내 안테나 수에 따른 $\mathrm{MF}$ 기반 셀 용량을 보여주고 있다. 전수 조사를 통한 최대 셀 용량은 그림 6 안의 네모 박스를 통해 보 여주고 있다. 그 값은 $2.869 \times 10^{8}$ 이며 해당 사용자 수 및 안테나 수는 동일하게 105 를 나타낸다. 식 (23)을 통한 최적 사용자 수 및 안테나 수는 105이 며, 식 (24)를 통한 최대 셀 용량은 $2.8689 \times 10^{8}$ 로 실험 결과와 동일함을 확인할 수 있다.

그림 7은 식 (23)의 최적 기지국 안테나 수 및 사용자 수를 적용한 셀 용량을 보여주고 있다. 기지 국의 송신 SNR을 증가시킴에 따라 $\mathrm{MF}$ 기반 셀 용 량은 식 (25)에서 분석한 이론적 결과와 같이 오목 


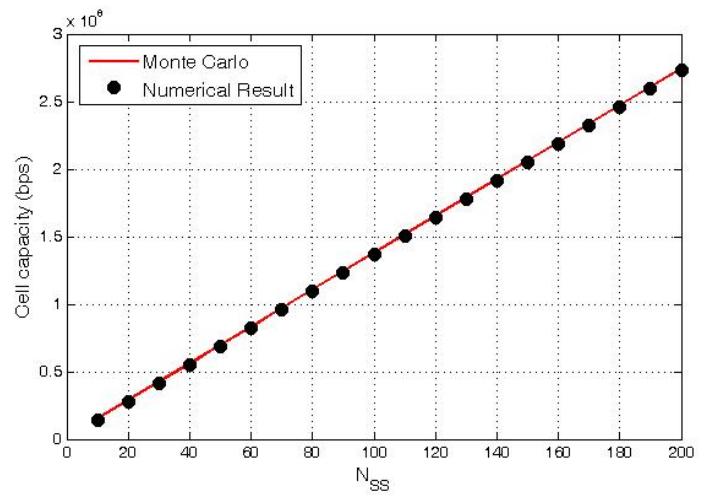

그림 8. 기지국의 송신 $\mathrm{SNR}$ 이 $0 \mathrm{~dB}$, 기지국 안테나 수 및 사용자 수가 모두 $N_{S S} / 2$ 인 경우의 $N_{S S}$ 값에 따른 셀 용량

Fig. 8. Cell capacity when transmit $\mathrm{SNR}=0 \mathrm{~dB}$ and $M$ $=K=N_{S S} / 2$

함수 형태이며 그 기울기가 0으로 수렴함을 확인할 수 있다. 또한 셀 용량은 식 (26)의 $N_{\mathrm{SS}} B T_{\mathrm{U}} / 4 T_{\mathrm{S}}=$ $4.9 \times 10^{4}$ 값에 수렴함을 그림 7을 통해 확인할 수 있다. 이는 식 (26)에서의 이론적 분석과 동일하며 이를 그림 7을 통해 실험적으로 확인할 수 있다.

그림 8은 기지국의 안테나 수 및 사용자의 수가 식 (23)에서 도출한 최적의 값을 가지고 있을 경우 $N_{S S}$ 값에 따른 $\mathrm{MF}$ 기반 셀 용량을 보여주고 있다. 적은 수의 $N_{S S}$ 값 영역에서도 실험을 통한 실험 값 과 추정한 수식은 어느 정도 유사한 결과를 나타내 고 있으며, 그 정확성은 $N_{S S}$ 값이 증가하면서 증가하 고 있음을 그림 8을 통해서 확인할 수 있다.

\section{VI. 결 론}

본 논문에서는 다중 사용자 massive MIMO 환 경에서의 $\mathrm{MF}$ 기반 셀 용량에 대한 분석을 진행하 였다. 특히, $\mathrm{MF}$ 를 사용하는 다중 사용자 massive MIMO 환경에서의 최적 안테나 수 및 사용자 수를 도출하였으며 이를 기반으로 한 최대 $\mathrm{MF}$ 셀 용량 을 수식적으로 도출하였다. 기지국 내 무한한 안테 나 수의 특성을 통해 평균 셀 용량을 기지국의 안 테나 수 및 사용자 수로 표현할 수 있다. 이러한 셀 용량은 기지국의 안테나 수 및 셀 내 사용자의 수에 대해 오목 함수이다. 셀 용량의 미분 과정을 통해 오목 함수임을 수식적으로 증명하였으며 실험 을 통해 이를 확인하였다. 또한 최적의 기지국 안테 나 수 및 사용자 수를 적용한 셀 용량에 대하여 무 한한 송신 $\mathrm{SNR}$ 을 가정한 상계 값을 수식적으로 도 출하였으며 실험적으로도 최적의 셀 용량은 송신 $\mathrm{SNR}$ 이 증가함에 따라 상계 값에 접근함을 확인하
였다. 본 논문에서는 파일럿을 통한 채널 추정이 완 벽하다고 가정하여 수식을 전개하였다. 향후 연구 과제로는 채널 추정 오류 (channel estimation error) 를 반영하여, 보다 실질적인 무선 통신 환경에서의 최적 기지국 안테나 수 및 사용자 수에 대한 연구 가 필요하다.

\section{References}

[1] A. J. Paulraj and T. Kailath, "Increasing capacity in wireless broadcast systems using distributed transmission/directional reception," U.S. Patent no. 5,345,599, 1994.

[2] I. E. Telatar, "Capacity of multi-antenna Gaussian channels," European Trans. Telecommun., vol. 10, no. 6, pp. 585-595, Nov./Dec. 1999.

[3] H. Ngo, E. Larsson, and T. Marzetta, "Energy and spectral efficiency of very large multiuser MIMO systems," IEEE Trans. Commun., vol. 61, no. 4, pp. 1436-1449, Apr. 2013.

[4] C. Peel, B. Hochwald, and A. Swindelhurst, "A vector-perturbation technique for near-capacity multiantenna multiuser communication-part I: channel inversion and regularization," IEEE Trans. Commun., vol. 53, no. 1, pp. 195-202, Jan. 2005.

[5] T. Yoo and A. Goldsmith, "On the optimality of multiantenna broadcast scheduling using zero-forcing beamforming," IEEE J. Select. Areas Commun., vol. 24, no. 3, pp. 528-541, Mar. 2006.

[6] C. Chae, S. Shim, and R. Heath, "Block diagonalized vector perturbation for multiuser MIMO systems," IEEE Trans. Commun., vol. 7, no. 11, pp. 4051-4057, Nov. 2008.

[7] H. Sung, S. Lee, and I. Lee, "Generalized channel inversion methods for multiuser MIMO systems," IEEE Trans. Commun., vol. 57, no. 11, pp. 3489-3499, Nov. 2009.

[8] F. Rusek, D. Persson, B. Lau, E. Larsson, T. Marzetta, O. Edfors, and F. Tufvesson, "Scaling up MIMO: opportunities and challenges with very large arrays," IEEE Signal Process. Mag., vol. 30, no. 1, pp. 40-60, Jan. 2013. 
[9] T. Marzetta, "Noncooperative cellular wireless with unlimited numbers of base station antennas," IEEE Trans. Wireless Commun., vol. 9, no. 11, pp. 3590-3600, Nov. 2010.

[10] C. Lee, C. Chae, T. Kim, S. Choi, and J. Lee, "Network massive MIMO for cell-boundary users: From a precoding normalization perspective," in Proc. IEEE Global Commun. Conf. (GLOBECOM), pp. 233-237, Anaheim, U.S.A., Dec. 2012.

[11] M. Jung, T. Kim, K. Min, Y. Kim, J. Lee, and S. Choi, "Asymptotic distribution of system capacity in multiuser MIMO systems with large number of antennas," in Proc. IEEE Veh. Technol. Conf. (VTC), pp. 1-5, Dresden, Germany, June 2013.

[12] B. Hochwald and S. Vishwanath, "Space-time multiple access: Linear growth in the sum rate," in Proc. 40th Annu. Allerton Conf. Commun., Control Comput., pp. 1-10, Monticello, U.S.A., Oct. 2002.

[13] S. Wagner, R. Couillet, M. Debbah, and D. Slock, "Large system analysis of linear precoding in correlated MISO broadcast channels under limited feedback," IEEE Trans. Inform. Theory, vol. 58, no. 7, pp. 4509-4537, July 2012.

[14] J, Jose, A. Ashikhmin, P. Whiting, and S. Vishwanath, "Channel estimation and linear precoding in multiuser multiple-antenna TDD systems," IEEE Trans. Veh. Technol., vol. 60, no. 5, pp. 2102-2116, June 2011.

[15] H, Ngo, E. Larsson, and T. Marzetta, "Analysis of the pilot contamination effect in very large multicell multiuser MIMO systems for physical channel models," in Proc. IEEE Int. Conf. Acoust., Speech, Signal Process. (ICASSP), pp. 3464-3467, Prague, Czech, May 2011.

[16] 3GPP, "Physical channels and modulation (Release 8)," 3GPP TS 36.211 v8.6.0, Mar. 2009.

[17] R. Corless, G. Gonnet, D. Hare, D. Jeffrey, and D. Knuth, "On the Lambert W-function,"
Advances in Computational Mathematics, vol. 5, pp. 329-359, 1996.

[18] M. Jung, Y. Kim, J. Lee, and S. Choi, "Optimal number of users in zero-forcing based multiuser MIMO systems with large number of antennas," J. Commun. Networks (JCN), vol. 15, no. 4, pp. 1-8, Aug. 2013.

정 민 채 (Minchae Jung)

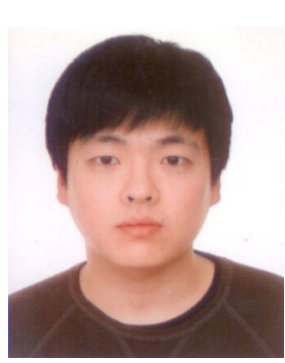

2008년 2월 연세대학교 전기 전자공학과 졸업

2008년 3월 현재 연세대학교 전기전자공학과 석박사 통 합과정

<관심분야> Massive MIMO,

Cognitive ratio network,

Body area network, D2D communication

\section{최 수 용 (Sooyong Choi)}

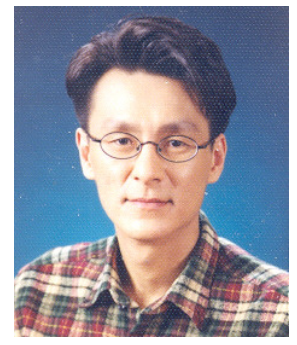

1995년 8월 연세대학교 전자 공학과 졸업

1997년 8월 연세대학교 전자 공학과 석사

2001년 8월 연세대학교 전자 공학과 박사

2002년 3월 2004년 8월

University of California, San Diego,

Postgraduate Researcher

2004년 9월 2005년 7월 Oklahoma State

University, Research Assistant Professor and

Researcher

2005년 9월 현재 연세대학교 전기전자공학과 부교 수

<관심분야> Massive MIMO, Interference management/coordination/cancellation/mitigation, Detection and estimation, Iterative receivers, 무 선 통신 시스템, Cognitive radios 